\title{
Discriminative expression of whole blood genes in HIV patients with latent and active TB in Ethiopia
}

Desta Kassa ${ }^{\mathrm{a}, \mathrm{b}^{*}}$, Leonie Ran ${ }^{\mathrm{b}}$, Wilco de Jager ${ }^{\mathrm{c}}$, Theo van den Broek ${ }^{\mathrm{c}}$, Ronald Jacobi $^{\text {b }}$, Muluberhan Mekonen ${ }^{\text {ad }}$, Tsehaynesh Messele ${ }^{\mathrm{a}}$, Mariëlle C Haks ${ }^{\mathrm{e}}$, Tom H. M. Ottenhoff ${ }^{\mathrm{e}}$, and Debbie van Baarle ${ }^{\mathrm{bf}}$

${ }^{\text {a }}$ HIV/AIDS and Tuberculosis Research Directorate, Ethiopian Public Health Institute (EPHI), Addis Ababa, Ethiopia

${ }^{\mathrm{b}}$ Department of Internal Medicine and Infectious Diseases, University Medical Center Utrecht, Utrecht, The Netherlands

${ }^{\mathrm{c}}$ Laboratory of Translational Immunology, Department of Immunology, University Medical Center Utrecht, Utrecht, The Netherlands

${ }^{\mathrm{d}}$ Axum University, Tigray, Ethiopia

${ }^{\mathrm{e}}$ Department of Infectious Diseases, Leiden University Medical Center, Leiden, The Netherlands

${ }^{\mathrm{f}}$ Current affiliation; Dept Immune mechanisms, center for Infectious Disease control, National Institute for Public Health and the Environment, Bilthoven, The Netherlands

* Corresponding author mailing address:

Ethiopian Public Health Institute (EPHI).

P.O. Box 1242, Addis Ababa, Ethiopia.

Phone: 251-0911-479212.

Fax: 251-0112-75-63-29.

E-mail: $\underline{\text { dkassa2003@gmail.com. }}$ 


\section{Abstract}

Background: Transcriptomic host biomarkers could assist in developing effective diagnostics, vaccines and therapeutics for tuberculosis (TB). However, different biomarkers may be discriminatory in different populations depending on the host and bacillary genetics and HIV infection, and need to be addressed.

Methods: The expression levels of 45 genes that are known to be involved in or affected by TB pathogenesis were analyzed using dual color Reverse Transcriptase Multiplex Ligation-dependent Probe Amplification (dcRT-MLPA) assay in whole blood of $106 \mathrm{HIV}$ positive individuals including active $\mathrm{TB}$ patients $\left(\mathrm{TB}^{+} \mathrm{HIV}^{+}\right.$, $\mathrm{n}=29)$, and non TB patients that are tuberculin skin test positive (TST+) $\left(\mathrm{TST}^{+} \mathrm{HIV}^{+}\right.$, $\mathrm{n}=26$ ), or TST negative ( $\mathrm{TST}^{-} \mathrm{HIV}^{+}, \mathrm{n}=51$ ).

Results: Between the two clinical groups $\left(\mathrm{TB}^{+} \mathrm{HIV}^{+}\right.$vs. TST $\left.\mathrm{HIV}^{+}\right) 8$ genes were differently expressed (CCL19, CD14, CD8A, FPR1, IL7R, CCL22, TNFRSF1A, and FCGR1A); between $\mathrm{TB}^{+} \mathrm{HIV}^{+}$vs. $\mathrm{TST}^{+} \mathrm{HIV}^{+}, 6$ genes (CD14, IL7R, TIMP2, CCL22, TNFRSF1A, and FCGR1A) were differently expressed. Since no difference in gene expression was revealed between $\mathrm{TST}^{+} \mathrm{HIV}^{+}$vs. $\mathrm{TST}^{-} \mathrm{HIV}^{+}$, we clustered both the $\mathrm{TST}^{+} \mathrm{HIV}^{+}$and $\mathrm{TST}^{-} \mathrm{HIV}^{+}$individuals as one group $\left(\mathrm{TST}^{+/} \mathrm{HIV}^{+}\right.$) and compared gene expression with $\mathrm{TB}^{+} \mathrm{HIV}^{+}$patients. Thus, the results revealed that the levels of five genes (CD8A, TIMP2, CCL22, FCGR1A and TNFRSF1A) were the most accurate single gene markers for differentiation between $\mathrm{TB}^{+} \mathrm{HIV}^{+}$and $\mathrm{TST}^{+/} \mathrm{HIV}^{+}$, with AUCs of $0.71,0.71,0.79,0.83$ and 0.73 , respectively. However, the combination of two genes (CCL22 +FCGR1A) and FCGR1A alone were the most accurate marker for differentiation between the two groups $\left(\mathrm{TB}^{+} \mathrm{HIV}^{+}\right.$and $\mathrm{TST}^{+/} \mathrm{HIV}^{+}$) with AUC of 0.85 and 0.83 , respectively.

Conclusions: We showed that five genes (CD8A, TIMP2, CCL22, FCGR1A and TNFRSF1A), specifically FCGR1A and CCL22 have the potential to discriminate active TB from non-active TB in HIV patients in Ethiopia and could be used to improve diagnostic tools for active TB in HIV patients, and to understand the pathogenesis of TB/HIV coinfection. 


\section{Introduction}

Tuberculosis (TB) remains a major global health threat next to HIV/AIDS. Factors which impair the success of TB control include lack of effective TB diagnostics [1]; lack of an effective vaccine [2]; the emergence of multi- and extensively drugresistant TB [MDR-and XDR-TB), and HIV infection [2]; limitations in anti-TB drugs and therapeutic regime (3]; social and economic factors (3]; and the nature of the spread of Mycobacterium tuberculosis (Mtb) infection and the complex host immune response [4].

TB biomarkers which can accurately predict reactivation of latent TB infection (LTBI), TB cure, eradication of latent TB, and efficacy of vaccine and drug therapy are important for development of effective diagnostic and therapeutic tools for TB. The discovery of specific and universal biomarkers for TB, therefore, is a priority of TB research [5, 6]. Biomarkers can be host and pathogen specific and can be determined at the cellular, protein or transcript level [7].

Several transcriptome studies have identified host gene signatures that are able to classify active from latent TB, including gene markers expressed by mononuclear cells [8], regulatory $\mathrm{T}$ cells [9], $\mathrm{B}$ and innate immunity pathways [10]. Overexpression of IFN-inducible transcripts in purified blood neutrophils from active TB patients as compared to healthy controls was also reported [11]

However, there are no universal biomarkers that can be utilized in the TB eradication program. The existence of at least six $M t b$ lineages that have co-evolved with and adapted to human populations [12], which may result in host resistance in specific human populations [13], suggests that different biomarkers may be discriminatory in different populations depending on the underlying $M t b$ epidemiology. Likewise, population-based differences in immune reactivity due to immune deviation by coinfections such as HIV and malaria may impact biomarker signatures in the local TB setting. Therefore, biomarker signatures for TB need to be investigated and validated across geographically and ethnically diverse populations $[14,15,16]$. 
In line with this, in a previous study, the expression pattern of 45 host genes implicated in TB pathogenesis was investigated in four sub-Saharan countries including Ethiopia, Malawi, South Africa, and The Gambia [17]. The study revealed differential expression of CXCR5, FCGR1A, CD3E and IL7R between TB cases and community controls in all sites regardless of HIV infection. More interestingly, FCGR1A was the most significant biomarker to classify active TB [17]. Furthermore, the study identified 11 genes (SEC14L1, CASP8, CXCR5, FCGR1A, CD3E, CD4, CD8A, IL7R, TGFB1, TGFBR2, BCL2) which were differently expressed between HIV negative active TB patients ( $\left.\mathrm{TB}^{+} \mathrm{HIV}^{-}\right)$and LTBI individuals $\left(\mathrm{HIV}^{-} \mathrm{TST}^{+}\right.$) [17]. Here we extend this previous study by including HIV infected individuals with latent and active TB, and aim to identify host gene markers that have discriminative potential for active TB infection in HIV infected individuals. 


\section{Materials and methods}

\section{Study population}

This cross-sectional study was performed from April 2007-February 2011 in Addis Ababa, Ethiopia. Adults (age 18-60 years) of both sexes who visited the halth falicties (St Peter Specialized Referral TB Hospital, Akaki and Kality Health centers) and who were naïve to ART and TB treatment were recruited consecutively after informed and written consent was sought. Diagnosis of active tuberculosis (TB) was based on both clinical and bacteriological evidences. At least two sputum smears stained by the Ziehil-Neelsen direct method were required to be microscopy positive for Acid Fast Bacilli (AFB) [18]. Except for TB patients, Tuberculin Skin Testing (TST) for tuberculin (PPD RT23, SSI, Copenhagen, Denmark) was done for all participants. A diameter of skin induration with $\geq 5 \mathrm{~mm}$ in HIV-infected individuals was graded as TST positive (TST+), and was considered as a LTBI [18]. The study participants were enrolled in 3 clinical groups: HIV positive active TB patients $\left(\mathrm{TB}^{+} \mathrm{HIV}^{+}\right)$, and asymptomatic non $\mathrm{TB}$ patients that are tuberculin skin test positive (TST+) $\left(\mathrm{TST}^{+} \mathrm{HIV}^{+}\right)$, and TST negative $\left(\mathrm{TST}^{-} \mathrm{HIV}^{+}\right)$.

At enrolment each participant was interviewed using a standard questionnaire and detailed clinical, anthropometric and demographic data were recorded by a clinician or a nurse. HAART was prescribed for eligible HIV patients and anti-TB treatment for all the TB cases free of charge as per the national guidelines. Antibiotic prophylaxis was also prescribed by the physician at enrolment or during the clinic follow-up visits [18].

\section{Laboratory Testing}

Twenty $\mathrm{ml}$ heparinized venous blood was collected from all subjects at diagnosis of HIV or TB and processed at the National HIV Referral Laboratory of the Ethiopian public health institute (EPHI), Addis Ababa. Laboratory examinations including blood CD4+ T cell counts and Plasma HIV RNA load were determined using BD 
FACSCalibur (Becton Dickinson, San Jose, USA), and NucliSens EasyQ NASBA diagnostic 2007/1 (Organon, Teknika), respectively, according to manufacturer's instruction.

\section{Dual color Reverse transcriptase Multiplex ligation-dependent probe amplification}

\section{(dcRT-MLP)}

mRNA was extracted from whole blood using PAXgene tubes and extraction kits according to the manufacturer's instructions (Qiagen, USA). The expression level (areas of each assigned peak in arbitrary units) of the 45 gene transcripts of interest that are associated with the host immune response components against $M t b$ infection including innate immunity, T-cell immunity and production of cytokines/chemokines $[19,20]$ was determined using dcRT-MLPA as previously described $[11,17]$. The expression level of each gene was normalized by adjustment to the glyceraldehyde3-phosphate dehydrogenase (GAPDH) housekeeping gene. Primers and probes were from Sigma-Aldrich Chemie (Zwijndrecht, the Netherlands) and MLPA reagents from MRC-Holland (Amsterdam, the Netherlands). Detailed characteristics of the panel of host gene targets included in this study was described previously [17].

\section{Statistical data analysis}

A descriptive analysis of the level of expression of each gene was conducted. Measured genes that had more than $25 \%$ of all measurements under the detection limit were excluded from further analysis unless significant correlations were found between the groups of interest. Thus, of the 45 host genes analyzed, the following genes were excluded: CTLA4, FOXP3, IL4, IL4d2, LAG3, MARCO, NCAM1, RAB33A, TNF, TNFRSF18, BPI, CCL13, CCL4, CCR7, CD163, CD19, FASLG, IFNG, IL-10, IL22RA1, RAB13, RAB24, IL2RA, LTF and SPP1. Of special interest, of these genes excluded from the analysis, six genes including MARCO, IL22RA1, BPI, CCL13, TNFRSF18, IL4, were not expressed at all in the study groups. 
Comparisons of quantitative variables between two groups were determined by Mann-Whitney U test and for more than two groups by Kruskal-Wallis test. Cut-off levels for differentiating between groups were determined by receiver operator characteristic (ROC) curve analysis using the " $\mathrm{R}$ " statistical programming language. The level of significance was at $\mathrm{P}<0.05$ and all $\mathrm{P}$ values were two-tailed. Bonferroni adjustment was performed for the measured genes in order to correct for inflation in type I error, setting the significance level from $\mathrm{p}<0.05$ to $<0.0025(=0.05 / 20)$. All the analyses were performed using Intercooled STATA version 10.0 (College Station, Texas, USA) and with the SPSS statistics software (IBM, version 20.0.0). Hierarchical cluster analysis was performed using Omniviz 6.1.2 (Instem scientific).

\section{Ethical approval}

This study was ethically approved institutionally, by the Scientific and Ethics Review Office (SERO), Ethiopian Public Health Institute (EPHI); and nationally by the National Health Research Ethics Review Committee (NHRERC), Ethiopian Ministry of Science and Technology Agency (ESTA). Thus, written and informed consent was obtained from each study participant. The consent procedure has been also reviewed and approved by the ethics committee (SERO) together with the study protocol. Finally, the signed consent was documented in a secured cabinet at EPHI. However, since no minors or children were enrolled in this study, we did not request and obtain informed consent from the next of kin, caretakers, or guardians. 


\section{Results}

\subsection{Demographic and clinical characteristics of the study participants}

A total of 106 participants $\left(29 \mathrm{~TB}^{+} \mathrm{HIV}^{+}, 26 \mathrm{TST}^{+} \mathrm{HIV}^{+}\right.$, and $51 \mathrm{TST}^{-} \mathrm{HIV}^{+}$) were included in this study. Mean (standard deviation, SD) age of the study participants was 31.6 (8.6) years, and 59 (39.6\%) were males. At diagnosis, the median CD4+ T cell count was lower in the $\mathrm{TB}^{+} \mathrm{HIV}^{+}$patients $(85$ cells $/ \mu \mathrm{l}$; Interquartile range (IQR):51-171; n=27), compared to $\mathrm{TST}^{+} \mathrm{HIV}^{+}$(280 cells/ $\mu 1 ; \mathrm{IQR}: 208-378 ; \mathrm{n}=22$ ),

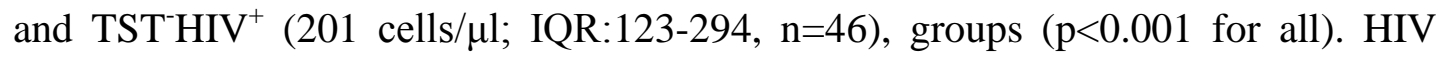
plasma RNA level was higher in $\mathrm{TB}^{+} \mathrm{HIV}^{+}$patients (59000 copies/ml; IQR: 2100015000; $\mathrm{n}=26$ ) compared to $\mathrm{TST}^{-} \mathrm{HIV}^{+}$(9300 copies/ml; IQR: 1100-53000 copies/ml; $\mathrm{n}=23$ ) and $\mathrm{TST}^{+} \mathrm{HIV}^{+}$individuals (21500 copies/ml; IQR: 4600-74000 copies/ml; $\mathrm{n}=46)$, ( $<<0.02$ for all). There was no significant difference in HIV plasma RNA load between $\mathrm{TST}^{-} \mathrm{HIV}^{+}$and $\mathrm{TST}^{+} \mathrm{HIV}^{+}$patients.

\subsection{Comparison of gene expression among $\mathrm{TB}^{+} \mathrm{HIV}^{+}, \mathrm{TST}^{+} \mathrm{HIV}^{+}$, and $\mathrm{TST}^{-}$ HIV $^{+}$groups}

We compared the gene expression level of 20 genes among the 3 clinical groups $\left(\mathrm{TB}^{+} \mathrm{HIV}^{+}, \mathrm{TST}^{+} \mathrm{HIV}^{+}\right.$, and $\left.\mathrm{TST}^{-} \mathrm{HIV}^{+}\right)$. Interestingly, the genes that showed significant differential expression among the 3 clinical groups were found to be CCL19, CD14, CD8A, IL7R, TIMP2, CCL22, TNFRST1A, FCGR1A ( $\mathrm{p}<0.05)$ (Table 1 and Fig 1).

Furthermore, gene expression patterns between two clinical groups were compared (Table 1). Between HIV infected individuals with and without active TB $\left(\mathrm{TB}^{+} \mathrm{HIV}^{+}\right.$ vs. TST $\mathrm{HIV}^{+}$), 12 genes were differently expressed (CCL19, CD14, CD8A, FPR1, IL7R, SEC14L1, TIMP2, CCL22, CD3E, TNFRSF1A, FCGR1A, and MMP9); whereas between HIV infected individuals with active TB and LTBI $\left(\mathrm{TB}^{+} \mathrm{HIV}^{+}\right.$vs. $\mathrm{TST}^{+} \mathrm{HIV}^{+}$), 7 genes (CD14, IL7R, TIMP2, CCL22, TNFRSF1A, FCGR1A, and MMP9) were differently expressed. 
However, since no difference in gene expression was revealed between HIV infected participants with and without LTBI $\left(\mathrm{TST}^{+} \mathrm{HIV}^{+}\right.$vs. $\left.\mathrm{TST}^{-} \mathrm{HIV}^{+}\right)$, we clustered the $\mathrm{TST}^{+} \mathrm{HIV}^{+}$and $\mathrm{TST}^{-} \mathrm{HIV}^{+}$individuals as one group $\left(\mathrm{TST}^{+/} \mathrm{HIV}^{+}\right)$, and a comparison was done with the $\mathrm{TB}^{+} \mathrm{HIV}^{+}$patients. As shown in Table 1, significant differences were found in the expression of CCL19, CD14, CD8A, CXCL10, FPR1, IL7R, SEC14L1, TIMP2, CCL22, MMP9, TNFRSF1A and FCGR1A genes $(p<0.05$ for all), where CD8A, IL7R, CD3E and SEC14L1 were expressed at higher levels in the $\mathrm{TST}^{+/} \mathrm{HIV}^{+}$, while the other genes were expressed at higher level in the $\mathrm{TB}^{+} \mathrm{HIV}^{+}$ patients.

Fig 1. Host mRNA genes which showed distinct expression among three clinical groups.

$\mathbf{T B}^{+} \mathbf{H I V}{ }^{+}=$HIV positive active TB patients; $\mathbf{T S T}^{+} \mathbf{H I V}^{+}=$HIV positive tuberculin skin test positive $(\mathrm{TST}+)$ participants; TST $^{-} \mathbf{H I V}^{+}=$HIV positive $\mathrm{TST}$ negative participants. dcRT-MLPA was done on RNA extracted from direct ex vivo whole blood from each study participant. Bar plots indicate mean expression values of the host mRNA genes. Horizontal lines in the boxes show median values, and boxes boundaries representing $25^{\text {th }}$ and $75^{\text {th }}$ percentiles. The responses were compared using a Mann-Whitney U test. P-values are shown on the horizontal lines. 


\subsection{The ability of five selected gene markers to discriminate active TB in HIV patients}

Furthermore, when Bonferroni adjustment was performed to see which genes were most relevant in differentiating active TB cases in HIV patients $\left(\mathrm{TB}^{+} \mathrm{HIV}^{+}\right.$vs. $\mathrm{TST}^{+/}$ $\mathrm{HIV}^{+}$), only CD14, IL7R, CD8A, TIMP2, CCL22, FCGR1A, and TNFRSF1A were found to be significantly differentially expressed.

Following this, we performed a logistic regression analysis to see which genes were most relevant in identifying these two groups $\left(\mathrm{TB}^{+} \mathrm{HIV}^{+}\right.$vs. $\left.\mathrm{TST}^{+-} \mathrm{HIV}^{+}\right)$. However CD14 and IL7R were not contributed any additional value to the genes already in our model (data not shown). Thus, expression levels of five genes (CD8A, TIMP2, CCL22, FCGR1A and TNFRSF1A) were the most accurate biomarkers that differentiated between the two groups $\left(\mathrm{TB}^{+} \mathrm{HIV}^{+}{ }^{\mathrm{vs}}\right.$. $\left.\mathrm{TST}^{+/} \mathrm{HIV}^{+}\right)$. The cut-off values and the respective accuracies (sensitivity, specificity, positive and negative predictive values and area under curve (AUC)) of these individual markers, the combination of these 5 markers, and of the two most relevant genes (CCL22, FCGR1A) for predicting active TB (TB+) status during HIV coinfection are shown in Table 2. 


\subsection{Analysis of gene expression using heat map analysis}

In depth hierarchical cluster analysis of the data from HIV positive participants with and without active $\mathrm{TB}\left(\mathrm{TB}^{+} \mathrm{HIV}^{+}\right.$vs. $\left.\mathrm{TST}^{-} \mathrm{HIV}^{+}\right)$displayed a distinct expression of 13 genes between the groups (Fig 2A). However, using the most stringent power of analysis ( $\mathrm{t}$ test pooled variance), the expression of four genes (FCGR1A, CCL22, TIMP2 and TNFRSF1A) was significantly increased in the $\mathrm{TB}^{+} \mathrm{HIV}^{+}$patients, while levels of IL7R and CD8A were increased in $\mathrm{TST}^{-} \mathrm{HIV}^{+}$patients (Fig 2B). Likewise, cluster analysis of the data from $\mathrm{TB}^{+} \mathrm{HIV}^{+}$and $\mathrm{TST}^{+} \mathrm{HIV}^{+}$participants, showed increased expression of FCGR1A and TIMP2 in the $\mathrm{TB}^{+} \mathrm{HIV}^{+}$patients, while levels of IL-7R and CD8A were increased in the $\mathrm{TST}^{+} \mathrm{HIV}^{+}$patients(Fig 2C).

In summary, the most important genes that showed significant differential among the 3 clinical groups $\left(\mathrm{TB}^{+} \mathrm{HIV}^{+}, \mathrm{TST}^{-} \mathrm{HIV}^{+}, \mathrm{TST}^{+} \mathrm{HIV}^{+}\right)$, using heat map analysis, were found to be FCGR1A, CCL22, TIMP2, TNFRST1A, IL7R and CD8A. 


\section{Discussion}

Understanding host gene expression patterns that are known to be involved in TB pathogenesis also in HIV coinfected, in HIV patients with latent and active TB could help to identify candidate gene biomarkers for $\mathrm{TB}$, as well as to understand the complex pathogenesis of TB and TB/HIV coinfection [11,21].

We identified 8 genes (CCL19, CD14, CD8A, FPR1, IL7R, CCL22, TNFRSF1A, and FCGR1A) that showed distinct expression between HIV patients with and without active $\mathrm{TB}\left(\mathrm{TB}^{+} \mathrm{HIV}^{+}\right.$vs. $\left.\mathrm{TST}^{-} \mathrm{HIV}^{+}\right)$. A previous study also has shown differential expression of FCGR1A, IL7R, CXCR5 and CD3E between HIV infected TB cases and community controls [17].

More importantly, in depth comparison of gene expression using logistic regression and cluster analysis, showed the ability of five genes (CD8A, TIMP2, CCL22, FCGR1A and TNFRSF1A) to distinguish active TB in HIV infected patients from HIV infected patients with and without LTBI (TST'HIV ${ }^{+}$and $\mathrm{TST}^{+} \mathrm{HIV}^{+}$) (Table 2). In support to our findings, a study performed in four Sub Saharan African countries (Ethiopia, Malawi, South Africa, and The Gambia) showed that the potential of Fc $\gamma$ Rs/ FCGR1A to discriminate active TB patients from latently infected individuals was, irrespective of HIV infection and ethnicity [17]. Others also reported the ability of FCGR1B, FCGR1A/CD64, and LTF [10] and FCGR1A, LTF, RAB13 [11] to discriminate active from latent TB.

The findings of our study may give insight into the host-bacilli-viral interrelations and TB/HIV pathogenesis. Several studies have shown associations between expression levels of host immune genes and pulmonary TB in Africa [22, 23]. Thus, the higher expression patterns of genes in HIV patients with active TB (Table 1), may indicate the influence of actively dividing bacilli ( $M t b$ antigen load) to trigger host gene expression. In support to our findings, a rapid change in blood gene expression profile which correlates with the clearance of the actively dividing bacilli in TB patients on therapy was reported [24]. 
On the other hand, the higher expression level of specific host mRNA genes in HIV patients with active TB, could indicate the activation of cellular immune responses in an attempt to control the Mtb infection [25], or the mechanism by which $M t b$ infection increases the progression of asymptomatic HIV infection to AIDS [26]. The differential expression of five immune genes (CD8A, TIMP2, CCL22, FCGR1A and TNFRSF1A) between the two groups $\left(\mathrm{TB}^{+} \mathrm{HIV}^{+}{ }^{-}\right.$vs. $\left.\mathrm{TST}^{+-} \mathrm{HIV}^{+}\right)$may suggest a role of these genes in the pathogenesis of TB during coinfection with HIV. Thus, the lower expression of the genes associated with T cells (CD3E, CD8A, and CD4) in $\mathrm{TB}^{+} \mathrm{HIV}^{+}$patients could be associated with the depletion of $\mathrm{T}$ cells from the periphery blood due to HIV infection and may indicate the mechanisms by which HIV infection aggravates TB disease progression.

Humoral immune responses are thought to be one of the host defense mechanisms against $M t b$ infection [27]. In the present study the levels of the B cell associated gene, the high-affinity $\mathrm{Fc}$ receptors (FcRs) for immunoglobulin (IGs) (FCGR1A/CD64), and the Matrix metalloproteinases (MMPs) members (TIMP2, MMP9), were elevated in $\mathrm{TB}^{+} \mathrm{HIV}^{+}$patients (Table 1and Fig 1). Fc $\gamma$ Rs, which are expressed solely by innate cells, bind the Fc portion of Igs and modulate immunity $[28,29]$. Studies showed that Fc $\gamma$ Rs regulate immune activation and susceptibility during $M t b$ infection by divergently affecting cytokine productions [28]. On the other hand, MMPs, which are secreted by monocytes, macrophages and activated lymphocytes, are known to degrade extracellular matrix such as collagen and proteoglycans [30]. It has been shown that infection of monocytes and macrophages with Mtb in vitro and in vivo induces MMP9 secretion [31]. The involvement of large amounts of MMP9 and TIMP2 in the pathogenesis and pathological changes of pulmonary TB (PTB) has also been reported [32,33]. Thus, our findings suggest further elucidating of the FCGR1A, MMP9 and TIMP2 in TB/HIV patients may provide new insights to the pathogenesis of TB, and to develop effective therapeutic and diagnostic tools for TB in HIV patients. 
T regulatory cells (Tregs) play a crucial role in the maintenance of self-tolerance and prevention of excessive responses against infection [34, 35]. In our study here, IL-7R was suppressed in HIV patients with active TB compared to those infected with latent $\mathrm{TB}$, as reported by others $[36,17]$. However, whether this could be beneficial for the host or for the pathogen needs further investigation.

Apoptosis of macrophages is an effective host strategy to control intracellular pathogen mycobacteria [37]. TNFR1 and TNFR2 are receptors for tumor necrosis factor- $\alpha$ (TNF- $\alpha)$ which is known to modulate apoptosis of infected macrophages [37]. Association of TNFR1 and TNFR2 with Pulmonary TB in Africa [38] has been reported. In agreement with others [39], we found higher expression of TNFRSF1A in $\mathrm{TB}^{+} \mathrm{HIV}^{+}$patients (Table 1). Although this needs further investigation, it may be beneficial to the host as it would be associated with diminished pathogen viability [40].

Chemokines play a protective role against $M t b$ infection by assisting cell migration and subsequently granuloma formation [20]. CCL22 is a chemotactic factor for Th2 cells [34], and for Tregs into the pleural space of patients with tuberculous pleurisy [41]. Thus, CCL22 could negatively modulate Th1 polarization and aggravates TB disease progression [42]. CCL9 which is the major beta-chemokine is known to facilitate the transport of $M t b$ from the lung to the lymph node via DCs; the accumulation of antigen-specific IFN- $\gamma$ producing $\mathrm{T}$ cells in the lung; and the development of the granuloma and control of mycobacteria [43]. Therefore, the higher expression level of CL22 and CCL9 in $\mathrm{TB}^{+} \mathrm{HIV}^{+}$patients in the present study, strongly indicate the role of CL22 and CCL9 in the pathogenesis of TB during HIV infection.

In summary, we identified several host genes from different families that are differentially expressed in HIV patients with active versus latent TB. More importantly, this study showed distinctive expression of five genes (CD8A, TIMP2, CCL22, FCGR1A and TNFRSF1A) between the two groups $\left(\mathrm{TB}^{+} \mathrm{HIV}^{+}\right.$vs. $\mathrm{TST}^{+/-}$ 
$\mathrm{HIV}^{+}$) which strongly indicates the prospect of these genes to discriminate active TB in HIV patients as well as their role in the pathogenesis of TB/HIV coinfection.

Limitations of the study

However, the fact that we did not include HIV negative active TB cases and healthy control groups in the study and that there is no golden standard definition of LTBI could be counted as limitations of the study. In addition, with the continuous refinement of the discriminative power of our large panel of potential genetic markers within the same study population, the possibility of overfitting the data is present, exaggerating minor fluctuations in the data. Although several of our identified genes are supported with previous reports, a different and larger cohort is needed to validate our findings. 


\section{Acknowledgements}

We would like to acknowledge all patients participating in this cohort study, all the study nurses involved, and the data clerk. This work, which is part of the longitudinal study entitled "Biomarkers of protective immunity against tuberculosis in the context of Human Immunodeficiency Virus/Acquired immunodeficiency Syndrome (HIV/AIDS) in Africa" [44], was supported by the Bill \& Melinda Gates Foundation through the Grand Challenges in Global Health Initiative (GCGH), grant no. 37772 . 


\section{References}

[1] Young DB, Perkins MD, Duncan K, Barry CE 3rd. Confronting the scientific obstacles to global control of tuberculosis. J. Clin. Investig. 2008; 118:1255-65

[2] Tom H. M. Ottenhoff and Stefan H. E. Kaufmann. Vaccines against Tuberculosis: Where Are We and Where Do We Need to Go? PLoS Pathog. 2012 May; 8(5): e1002607.

[3] Colditz G, Brewer T, Berkey C, Wilson M, Burdick E, Fienberg H and Mosteller F. Efficacy of BCG vaccine in the prevention of tuberculosis. Met-analyisi of the published literature. JAMA 1994; 271: 698-702

[4] O'Garra A, Redford PS, McNab FW, Bloom CI, Wilkinson RJ, Berry MP. The immune response in tuberculosis. Annu Rev Immunol. 2013;31:475-527

[5] Wallis RS, Pai M, and Menzies D. Biomarkers and diagnostics for tuberculosis: progress, needs and translation in to practice. Lancet. 2010; 375:1920-37

[6] Gerhard W, Katharina R, Willem H, Thomas JS, and Alimuddin Z. Immunological biomarkers of tuberculosis. Natur Reviews: Immunol. 200; 11: 343354

[7] Engwegen JY, Helgason HH, Cats A, Harris N, Bonfrer JM, Schellens JH, Beijnen JH. Identification of serum proteins discriminating colorectal cancer patients and healthy controls using surface-enhanced laser desorption ionisation-time of flight mass spectrometry. World J Gastroenterol. 2006;12(10):1536-44.

[8] Jacobsen M, Repsilber D, Gutschmidt A. Candidate biomarkers for discrimination between infection and disease caused by Mycobacterium tuberculosis. J Mol Med. 2007; 85: 613-621.

[9] Maertzdorf J, Repsilber D, Parida SK, Stanley K, Roberts T, and Black G. Human gene expression profiles of susceptibility and resistance in tuberculosis. Genes Immun. 2011;12: 15-22. 
[10] Joosten SA, Goeman JJ, Sutherland JS et al. Identification of biomarkers for tuberculosis disease using a novel dual-color RT-MLPA assay. Genes Immun. 2012; 13: $71-82$.

[11] Berry MP, Graham CM, McNab FW, Xu Z, Bloch SA, Oni T, Wilkinson KA, Banchereau R, Skinner J, Wilkinson RJ, Quinn C, Blankenship D, Dhawan R, Cush JJ, Mejias A, Ramilo O, Kon OM, Pascual V, Banchereau J, Chaussabel D, O'Garra A. An interferon-inducible neutrophil-driven blood transcriptional signature in human tuberculosis. Nature. 2010 Aug 19;466(7309):973-7.

[12] Gagneux S, and Small PM. Global phylogeography of Mycobacterium tuberculosis and implications for tuberculosis product development. Lancet Infect Dis. 2007; 7(5):328-37.

[13] Van Crevel R, Ottenhoff THM, and Van der Meer WM. Innate immunity to Mycobacterium tuberculosis. Clin Microbiol Rev. 2009; 15: 294-309

[14] Ottenhoff HM, Jerrold J. Ellner B, and Kaufmann SH. Ten challenges for TB biomarkers. Tuberculosis. 2012; 92S1: S17-S20

[15] Doherty T Mk, Wallis R S and Zumla A. Biomarkers of Disease Activity, Cure, and Relapse in tuberculosis. Clin Chest Med. 2009; 783-796

[16] Walzl G, Ronacher K, Hanekom W, Scriba TJ, Zumla A. Immunological biomarkers of tuberculosis. Nat Rev Immunol. 2011;11(5):343-54.

[17] Sutherland JS, Lalor MK, Black GF, Ambrose LR, Loxton AG, Chegou NN, Kassa D, Mihret A, Howe R, Mayanja-Kizza H, Gomez MP, Donkor S, Franken K, Hanekom W, Klein MR et al. Analysis of host responses to Mycobacterium tuberculosis antigens in a multi-site study of subjects with different TB and HIV infection states in sub-Saharan Africa. PLoS One. 2013;8(9):e74080.

[18] Federal Ministry of Hearlth (FMOH). Guidline for clinical and programatic managment of TB, TB/HIV and Leprosy in Ethiopia. Fifth Edition. March, 2013.

[19] Flynn J: Immunology of tuberculosis and implications in vaccine development. Tuberculosis. 2004; 4:93-101. 
[20] Zuniga J, Torees-Garicia D, Santos-Mendoza T, Rodriguez-Reyna T, Granados $\mathbf{J}$, and Yunis EJ. Cellular and Humoral mechanisms involved in the Control of Tuberculosis. Clin Dev Immunol. 2012;2012:193923.

[21] Bonecini-Almeida MG, Ho JL, Boechat N, Huard RC, Chitale S, and Doo H. Down-modulation of lung immune responses by interleukin-10 and transforming growth factor beta (TGF-beta) and analysis of TGF-beta receptors I and II in active tuberculosis. Infect Immun. 2004; 72: 2628-2634.

[22] Buijtels PC, van de Sande WW, Parkinson S, Petit PL, van der Sande MA, van $\mathrm{SD}$, Verbrugh HA, van BA. Polymorphism in CC-chemokine ligand 2 associated with tuberculosis in Zambia. Int J Tuberc Lung Dis. 2008; 12:1485-1488

[23] Moller M, Nebel A, Valentonyte R, van Helden PD, Schreiber S, Hoal EG Investigation of chromosome 17 candidate genes in susceptibility to TB in a South African population. Tuberculosis (Edinb ). 2009; 89:189-94

[24] Cliff JM, Lee JS, Constantinou N, Cho JE, Clark TG, Ronacher K, King EC, Lukey PT, Duncan K, Van Helden PD, Walzl G, Dockrell HM. Distinct phases of blood gene expression pattern through tuberculosis treatment reflect modulation of the humoral immune response. J Infect Dis. 2013;207(1):18-29.

[25] Oh MD, Park SW, and Kim HB. Spectrum of opportunistic infections and malignancies in patients with human immunodeficiency virus infection in South Korea. Clin Infect Dis. 1999; 29:1524-8.

[26] Siawaya D, Ruhwald M, Eugen-Olsen J, Walzl G (2007). Correlates for disease progression and prognosis during concurrent HIV/TB infection: Review. Int J Infect Dis. 2007;11(4):289-99.

[27] Olivares N, Puig A, Aguilar D. et al. Prophylactic effect of administration of human gamma globulins in a mouse model of tuberculosis," Tuberculosis. 2009; 89 (3):218- 220 . 
[28] Maglione PJ, Xu J, Casadevall A, Chan J. Fc gamma receptors regulate immune activation and susceptibility during Mycobacterium tuberculosis infection. J Immunol. 2008;180(5):3329-38.

[29] Guilliams M, Bruhns P, Saeys Y, Hammad H, Lambrecht BN. The function of Fc $\gamma$ receptors in dendritic cells and macrophages. Nat Rev Immunol. 2014 ;14(2):94108.

[30] Sheen P, O'Kane CM, Chaudhary K, Tovar M, Santillan C, Sosa J, and Caviedes L. High MMP9 activity characterises pleural tuberculosis correlating with granuloma formation. Eur. Respir. J. 2009;33: 134-141.

[31] Price NM, Farrar J, Tran TT, Nguyen TH, Tran TH, and Friedland JS. Identification of a matrix-degrading phenotype in human tuberculosis in vitro and in vivo. J Immunol. 2001;166: 4223-4230.

[32] De Groote MA, Nahid P, Jarlsberg L, Johnson JL, Weiner M, Muzanyi G, Janjic N, Sterling DG, Ochsner UA: Elucidating novel serum biomarkers associated with pulmonary tuberculosis treatment. PLoS One. 2013, 8(4):e61002

[33] Taylor JL, Hattle JM, Dreitz SA, Troudt JM, Izzo LS, Basaraba RJ, Orme IM, Matrisian LM, Izzo AA: Role for matrix metalloproteinase 9 in granuloma formation during pulmonary Mycobacterium tuberculosis infection. Infect Immun 2006, 74(11):6135-6144.

[34] Imai T, Nagira M, Takagi S, Kakizaki M, Nishimura M, Wang J, Gray PW, Matsushima K, Yoshie O. Selective recruitment of CCR4-bearing Th2 cells toward antigen-presenting cells by the $\mathrm{CC}$ chemokines thymus and activation-regulated chemokine and macrophage-derived chemokine. Int Immunol. 1999; 11(1):81-8.

[35] Remus N, El BJ, Fieschi C, Feinberg J, Quintin T, Chentoufi M, Schurr E, Benslimane A, Casanova JL, Abel L. Association of IL12RB1 polymorphisms with pulmonary tuberculosis in adults in Morocco. J Infect Dis. 2004; 190:580-587 
[36] Boer MC1, Joosten SA1, Ottenhoff TH1. Regulatory T-Cells at the Interface between Human Host and Pathogens in Infectious Diseases and Vaccination. Front Immunol. 2015; 6: 217.

[37] Rodrigues MF, Alves CC, Figueiredo BB, Rezende AB, Wohlres-Viana S, Silva VL, Machado MA, Teixeira HC. Tumour necrosis factor receptors and apoptosis of alveolar macrophages during early infection with attenuated and virulent Mycobacterium bovis. Immunology. 2013 ;139(4):503-12.

[38] Cooke GS, Campbell SJ, Sillah J, Gustafson P, Bah B, Sirugo G, Bennett S, McAdam KP, Sow O, Lienhardt C, Hill AV. Polymorphism within the interferongamma/receptor complex is associated with pulmonary tuberculosis. Am J Respir Crit Care Med. 2006; 174:339-343

[39] Placido R, Mancino G, Amendola A, Mariani F, Vendetti S, Piacentini M, Sanduzzi A, Bocchino ML, Zembala M, and Colizzi V. Apoptosis of human monocytes/macrophages in Mycobacterium tuberculosis infection. J. Pathol. 1997; $181: 31-38$.

[40] Behar SM, Martin CJ, Booty MG, Nishimura T, Zhao X, Gan HX, Divangahi M, Remold HG. Apoptosis is an innate defense function of macrophages against Mycobacterium tuberculosis. Mucosal Immunol. 2011 ;4(3):279-87.

[41] Wu C, Zhou Q, Qin XJ, Qin SM, Shi HZ. CCL22 is involved in the recruitment of $\mathrm{CD} 4+\mathrm{CD} 25$ high $\mathrm{T}$ cells into tuberculous pleural effusions. Respirology. 2010;15(3):522-9.

[42] Galli G, Chantry D, and Annunziato F. Macrophage-derived chemokine production by activated human $\mathrm{T}$ cells in vitro and in vivo: preferential association with the production of type 2 cytokines. Eur. J. Immunol. 2000; 30:204-210.

[43] Khader SA, Rangel-Moreno J, Fountain JJ, Martino CA, Reiley WW, Pearl JE, Winslow GM, Woodland DL, Randall TD, Cooper AM. In a murine tuberculosis 
model, the absence of homeostatic chemokines delays granuloma formation andprotective immunity. J Immunol. 2009;183(12):8004-14.

[44] Kaufmann, S.H.E. and S.K. Parida. 2008. Tuberculosis in Africa: Learning from Pathogenesis for Biomarker Identification. Cell Host Microbe. 4 (3): 219-228. 
Table 1. Analysis of gene expression in HIV infected individuals with latent and active TB

\begin{tabular}{|c|c|c|c|c|c|}
\hline Gene & $\begin{array}{l}\mathrm{TB}^{+} \mathrm{HIV}^{+} \\
(\mathrm{n}=29)\end{array}$ & $\begin{array}{l}\mathrm{TST}^{+} \mathrm{HIV}^{+} \\
(\mathrm{n}=26)\end{array}$ & TST HIV $^{+}(n=51)$ & p-value ${ }^{\S}$ & $\begin{array}{l}\text { TST }^{+/-} \text {HIV }^{+} \\
(\text {combined) }(\mathbf{n}=77)\end{array}$ \\
\hline BCL2 & $380.7 \pm 338.0$ & $394.3 \pm 371.2$ & $466.3 \pm 347.5$ & 0.182 & $442.0 \pm 354.9$ \\
\hline CXCR5 & $534.3 \pm 290.7$ & $494.6 \pm 412.0$ & $626.5 \pm 449.7$ & 0.437 & $582.0 \pm 439.1$ \\
\hline CASP8 & $4088.1 \pm 1840.1$ & $3575.3 \pm 2294.8$ & $4158.3 \pm 1964.7$ & 0.406 & $3961.4 \pm 2085.4$ \\
\hline CCL19 & $220 \pm 201.2$ & $131.8 \pm 186.2$ & $133.6 .6 \pm 163.8^{*}$ & 0.017 & $133 \pm 170.4^{*}$ \\
\hline CD14 & $2173.8 \pm 1124.5$ & $1682.8 \pm 1694.7^{*}$ & $1501.5 \pm 1281.5^{*}$ & 0.008 & $1562.7 \pm 1425.7 * *$ \\
\hline CD4 & $494.1 \pm 473.4$ & $745.8 \pm 665.3$ & $833.0 \pm 844.6$ & 0.166 & $803.5 \pm 785.2$ \\
\hline CD8A & $1994.3 \pm 1043.0$ & $2901.9 \pm 1604.1$ & $3998.5 \pm 2854.6^{* *}$ & 0.001 & $3637.8 \pm 2554.4^{* *}$ \\
\hline CXCL10 & $3656.9 \pm 3954.0$ & $2456.1 \pm 2967.2$ & $2037.1 \pm 2278.6$ & 0.123 & $2176.8 \pm 2516.5^{*}$ \\
\hline FPR1 & $10666.9 \pm 5048.6$ & $8891.8 \pm 5209.4$ & $8784.8 \pm 6222.2 *$ & 0.094 & $8820.9 \pm 5865.2 *$ \\
\hline IL7R & $1330.4 \pm 1117.6$ & $2281.4 \pm 2003.4^{*}$ & $2582.9 \pm 1953.4^{* *}$ & 0.005 & $2481.1 \pm 1962.5^{* *}$ \\
\hline SEC14L1 & $8148.5 \pm 2343.7$ & $9139.6 \pm 2358.0$ & $9512.4 \pm 3207.3^{*}$ & 0.118 & $9389.8 \pm 2944.2 *$ \\
\hline TGFBR2 & $1080.3 \pm 363.4$ & $957.1 \pm 643.8$ & $1180.8 \pm 652.5$ & 0.321 & $1105.2 \pm 654.0$ \\
\hline TIMP2 & $9869.0 \pm 4374.4$ & $6650.6 \pm 3651.8 *$ & $6929.7 \pm 4149.0^{*}$ & 0.005 & $6835.5 \pm 3966.1 * *$ \\
\hline CCL22 & $693.5 \pm 297.1$ & $396.4 \pm 329.9 * *$ & $341.3 \pm 318.1 * *$ & 0.000 & $359.4 \pm 320.9 * *$ \\
\hline CD3E & $2241.7 \pm 1799.5$ & $3209.2 \pm 2788.3$ & $3345.9 \pm 2424.2 *$ & 0.074 & $3299.7 \pm 2535.3 *$ \\
\hline TGFB1 & $1086.6 \pm 380.3$ & $1054.1 \pm 590.2$ & $1087.2 \pm 640.1$ & 0.943 & $1076.0 \pm 620.0$ \\
\hline TNFRSF1A & $3316.2 \pm 1319.4$ & $2131.3 \pm 1113.9 * *$ & $2352.5 \pm 1405.2 * *$ & 0.001 & $2279.7 \pm 1313.1 * *$ \\
\hline TNFRSF1B & $984.7 \pm 576.1$ & $851.2 \pm 493.8$ & $787.7 \pm 642.8$ & 0.174 & $808.6 \pm 595.3$ \\
\hline FCGR1A & $707.3 \pm 355.3$ & $376.8 \pm 510.7 * *$ & $221.6 \pm 312.0 * *$ & 0.000 & $274.0 \pm 394.0 * *$ \\
\hline MMP9 & $418.8 \pm 576.6$ & $176.1 \pm 463.9^{*}$ & $149.6 \pm 317.6^{*}$ & 0.209 & $176.1 \pm 370.3 * *$ \\
\hline
\end{tabular}

$\mathbf{T B}^{+} \mathbf{H I V}{ }^{+}=$HIV positive active TB patients; $\mathbf{T S T}^{+} \mathbf{H I V} \mathbf{V}^{+}=$HIV positive tuberculin skin test positive(TST+) individuals; TST$^{-} \mathbf{H I V}^{+}=$HIV positive $\mathrm{TST}$ negative individuals; TST $^{+-} \mathbf{H I V}^{+}=$both $\mathrm{TST}^{+} \mathrm{HIV}^{+}$and $\mathrm{TST}^{-} \mathrm{HIV}^{+}$combined

${ }^{\S}$ Data was tested with Kruskal-Wallis test among the three clinical groups $\left(\mathbf{T B}^{+} \mathbf{H I V}^{+}\right.$, TST $^{+} \mathbf{H I V}^{+}$, TST $^{-} \mathbf{H I V}^{+}$); and with by Mann-Whitney U test for two individual groups. ${ }^{*} \mathrm{p}<0.05$ and ${ }^{* *} \mathrm{p}<0.0025$ for $\mathbf{T S T}^{+} \mathbf{H I V}^{+}$, $\mathbf{T S T}^{-} \mathbf{H I V}^{+}$and $\mathbf{T S T}^{+/-}$ $\mathrm{HIV}^{+}$clinical groups as compared to the $\mathrm{TB}^{+} \mathrm{HIV}^{+}$group. Gene expression data is shown as mean \pm standard deviation. 
Table 2. Abilities of the top host genes to predict active TB status in HIV infected TB suspects

\begin{tabular}{|c|c|c|c|c|c|c|}
\hline Host gene & $\begin{array}{l}\text { Level in } \\
\text { TB }^{+} \mathbf{H I V}^{+} \\
(\mathbf{n}=29)\end{array}$ & $\begin{array}{l}\text { Level in } \\
\text { TST }^{+/-} \\
\text {HIV }^{+} \\
(\mathbf{n}=77)\end{array}$ & AUC & Cut-off & Sens & Spec \\
\hline CD8A & $\begin{array}{l}1994.3 \pm \\
1043.0\end{array}$ & $\begin{array}{l}3637.8 \\
\pm 2554.4\end{array}$ & 0.711 & $<11.25$ & 75.9 & 67.5 \\
\hline TIMP2 & $\begin{array}{l}9869.0 \pm \\
4374.4\end{array}$ & $\begin{array}{l}6835.5 \pm \\
3966.1\end{array}$ & 0.706 & $>12.95$ & 69.0 & 63.6 \\
\hline CCL22 & $\begin{array}{l}693.5 \pm 29 \\
7.1\end{array}$ & $\begin{array}{l}359.4 \pm \\
320.9\end{array}$ & 0.788 & $>8.95$ & 75.9 & 71.4 \\
\hline FCGR1A & $\begin{array}{l}707.3 \pm \\
355.3\end{array}$ & $\begin{array}{l}274.0 \\
\pm 394.0\end{array}$ & 0.826 & $>8.85$ & 79.3 & 80.5 \\
\hline $\begin{array}{l}\text { TNFRSF1 } \\
\text { A }\end{array}$ & $\begin{array}{l}3316.2 \pm \\
1319.4\end{array}$ & $\begin{array}{l}2279.7 \pm \\
1313.1\end{array}$ & 0.725 & $>11.25$ & 79.3 & 61.0 \\
\hline $\begin{array}{l}\text { All } 5 \text { genes } \\
\text { CCL22 } \\
\text { +FCGR1A }\end{array}$ & & & $\begin{array}{l}0.861 \\
0.852\end{array}$ & $\begin{array}{l}0.748 \\
0.760\end{array}$ & $\begin{array}{l}82.8 \\
82.8\end{array}$ & $\begin{array}{l}72.7 \\
76.6\end{array}$ \\
\hline
\end{tabular}

Data is shown as mean \pm standard deviations . AUC $=$ area under curve; Sens = sensitivity; Spec $=$ Specificity 

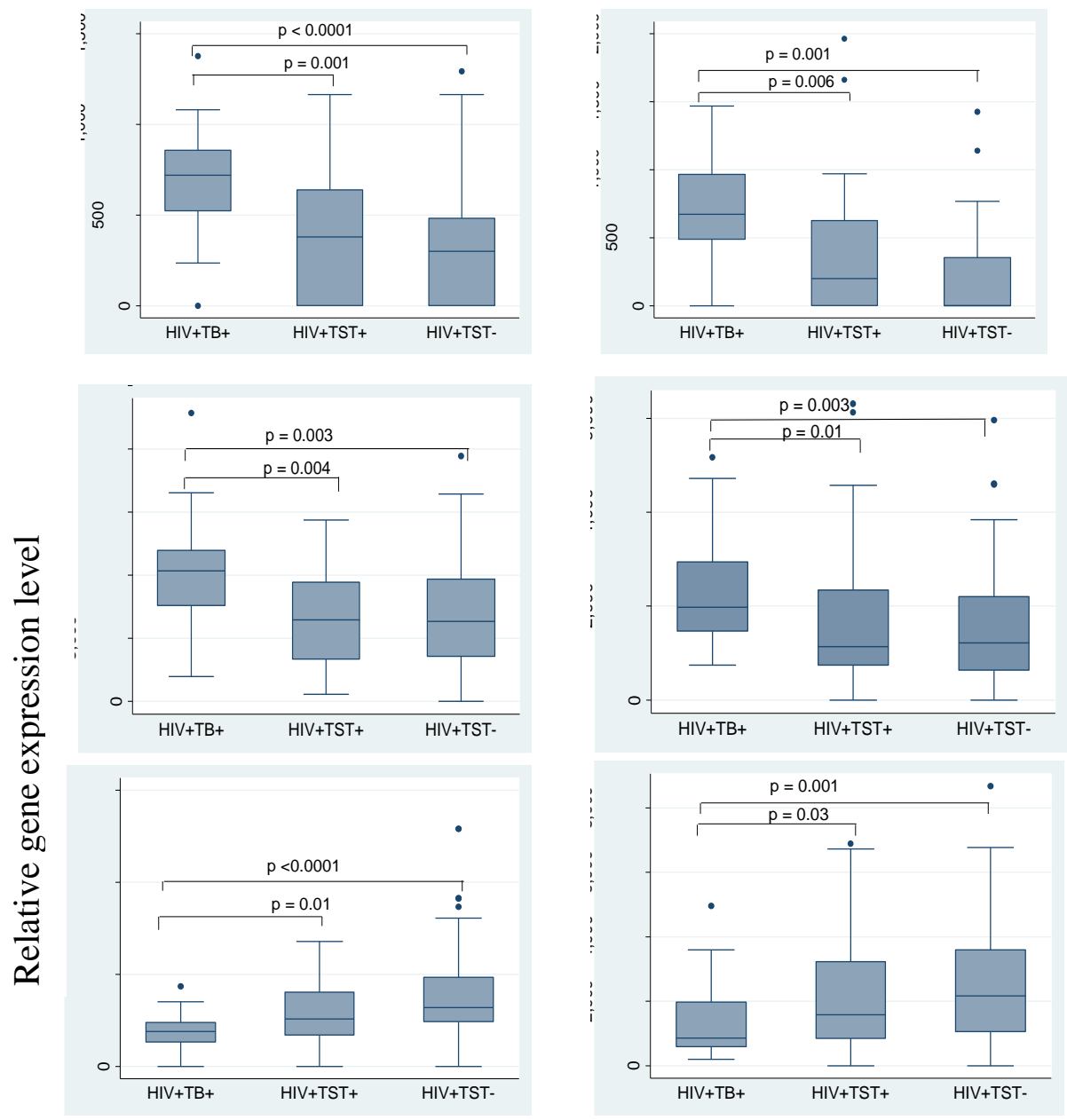

Fig 1. Host mRNA genes which showed distinct expression among three clinical groups.

$\mathbf{T B}^{+} \mathbf{H I V}{ }^{+}=$HIV positive active TB patients; $\mathbf{T S T}^{+} \mathbf{H I V}^{+}=$HIV positive tuberculin skin test positive $(\mathrm{TST}+)$ participants; $\mathbf{T S T}^{-} \mathbf{H I V}^{+}=$HIV positive $\mathrm{TST}$ negative participants. dcRT-MLPA was done on RNA extracted from direct ex vivo whole blood from each study participant. Bar plots indicate mean expression values of the host mRNA genes. Horizontal lines in the boxes show median values, and boxes boundaries representing $25^{\text {th }}$ and $75^{\text {th }}$ percentiles. The responses were compared using a Mann-Whitney U test. P-values are shown on the horizontal lines. 


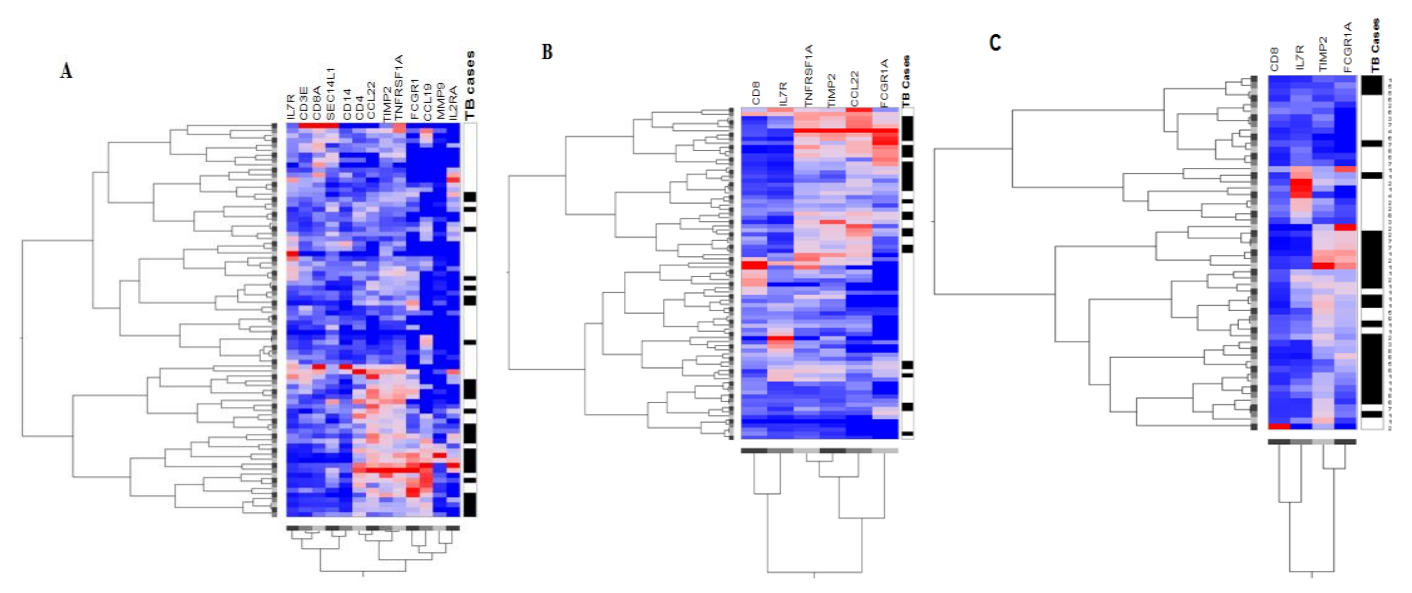

Fig 2: Heat map analysis of gene expression among three clinical groups.

(A) HIV positive active $\mathrm{TB}$ cases $\left(\mathrm{TB}^{+} \mathrm{HIV}^{+}\right.$) (black box) and HIV positive tuberculin skin test negative $\left(\mathrm{TST}^{-} \mathrm{HIV}^{+}\right.$) (white box), (B) $\mathrm{TB}^{+} \mathrm{HIV}^{+}$(black box), and $\mathrm{TST}^{-} \mathrm{HIV}^{+}$(white box), and (C) $\mathrm{TB}^{+} \mathrm{HIV}^{+}$(black box), and HIV positive tuberculin skin test positive $\left(\mathrm{TST}^{+} \mathrm{HIV}^{+}\right)$(white box). 